\title{
ISOLATION OF B-ACTIN PROMOTER DERIVED FROM WALKING CATFISH (Clarias batrachus)
}

\author{
Ibnu Dwi Buwono 1)*, Nono Carsono2), Yuniar Mulyani1), \\ Mochamad Untung Kurnia Agung1) \\ 'Faculty of Fisheries and Marine Sciences, University of Padjadjaran \\ 2Faculty of Agriculture, University of Padjadjaran \\ *e-mail : ibnudw1@yahoo.com
}

\begin{abstract}
Fish growth improvement as economic traits can be solved through fish transgenic production. Growth hormone gene is inserted into transgenic vector construction to over-express fish growth. The promoter as a part of the expression vector has an important role in its regulation. The use of promoter which is derived from mammalian or virus (such as CMV/ cytomegalovirus) in the expression vector, in specific goal as food material, has customer resistant rather than a promoter which is derived from in-sibling species. Beside of it, transgene expression level when using in-sibling promoter showed higher than using mammalian or viral promoter. The $\beta$-actin promoter is screened from walking catfish pituitary genome DNA using primers: pBA-cy-F (5'GTGWGTGACGCYGGACCAAATC-3') as forward primer and pBA-cy-R (5'CCATRTCRTCCCAGTTGGTSACAAT-3') as reverse primer, produced an amplicon of $1,7 \mathrm{~kb}$ in length. Sequence analysis using TF Bind ${ }^{T M}$ indicated transcription factor elements: TATA box, CCAAT box, enhancer (CAAT), and CarGG (CAAATGG) motif. This result showed that promoter which is obtained from this research is useful in construction of all catfish growth hormone vector expression catfish transgenic production.
\end{abstract}

Keywords: $\beta$-actin promoter, growth hormone, expression vector, walking catfish (Clarias batrachus)

\section{INTRODUCTION}

Generally, the gene construct contains an promoter sequence, protein encoding gene and polyadenilation signal that is used for fish transgenic production. The expression regulator element which is inserted and can active in many types of fish tissues is $\beta$-actin promoter (Fletcher et al. 2011). The $\beta$-actin promoter is effective to promote fish transgenic expression . These promoter was also evidence to regulate the expression of the gene encoding 6desaturase enzyme in zebrafish (Alimuddin et al. 2005) and the gene encoding growth hormone in tilapia (Kobayashi et al. 2007).

The use of promoters which are derived from mammalian or virus is known has lower expression level rather than promoter which is derived from in-sibling species (Alam et al., 1996). In zebrafish transgenic production, the viral promoter (cytomegalovirus / CMV) has lower expression level compared with $\beta$-actin promoter from zebrafish, because not all of cisacting elements of viral promoter are recognized by trans-acting elements of zebrafish (Alimuddin 2003). The use of in-sibling species promoters in the construction of transgenic fish also sounds safely in customers perception rather than when using mammalian or viral promoters (Maclean \& Laight 2000). 
$\beta$-actin promoter has known to be active within the same, in-sibling or different species of origin source of promoter, like within tilapia (Kobayashi et al. 2007), catfish (Ath-thar 2007), and zebrafish (Alimuddin et al. 2005). This promoter is an ubiquitous regulator in fish transgenic development (Higashijima et al. 1997). The $\beta$-actin is a highly conserved protein which is involved in cell motility, structure, and integrity. B-actin promoter sequence walking catfish (C. batrachus) were isolated using yellow catfish $\beta$-actin promoter primer (Ge et al. 2012), useful for the production of growth hormone expression vector "all catfish" of construction of transgenic fish.

\section{MATERIALS AND METHODS}

\section{Isolation of Genomic DNA of Walking Catfish}

The genomic DNA was isolated using Wizard genomic purification kit (Promega) from pituitary gland of C. batrachus. The gland was placed in a $1.5 \mathrm{~mL}$ microtube, grinded and added the chilled $300 \mu \mathrm{l}$ nuclei lysis solution. The solution was then incubated on waterbath at $65{ }^{\circ} \mathrm{C}$ for 15 minutes, and then $1.5 \mu \mathrm{l}$ RNase was added to the tube and be incubated on waterbath at $37{ }^{\circ} \mathrm{C}$ for 20 minutes.

As the next step, $100 \mu \mathrm{l}$ of protein precipitation solution was added into the tube, and placed it on-ice for 5 minutes. The tube was then centrifuged at $12000 \mathrm{rpm}$ for 4 minutes. The supernatant was transferred into a new sterile $1.5 \mathrm{~mL}$ microtube, which has contained $300 \mu \mathrm{l}$ of isopropanol, and mixed it with gently inverting. The tube was then centrifuged at 12000 rpm for 1 minute. The supernatant removed and $300 \mu \mathrm{l}$ of chilled etanol $70 \%$ added into the tube and it be centrifuged $12000 \mathrm{rpm}$ for 1 minute. The ethanol is removed and lets the tube dry. The DNA then be rehydrated by putting $50 \mu \mathrm{l}$ of rehydration solution into the tube and incubate it on waterbath at $65^{\circ} \mathrm{C}$ for 1 hour.

\section{PCR Amplification}

The $\beta$-actin promoter sequence was amplified by PCR method using primers: pBA-cy-F (5'GTGWGTGACGCYGGACCAAATC-3') as forward primer and pBA-cy-R (5'CCATRTCRTCCCAGTTGGTSACAAT-3') as reverse primer (Ge et al. 2012). The PCR cocktails contained: $12.5 \mu \mathrm{l}$ of fast start pcr master mix (Roche); $1.5 \mu \mathrm{l}$ of pBAYc-F $(10 \mu \mathrm{M}) ; 1.5 \mu \mathrm{l}$ of PBAYc-R $(10 \mu M) ; 2.5 \mu$ l of genomic DNA and $7 \mu$ l of nuclease free water.

The PCR program used was: $95^{\circ} \mathrm{C}$ for 5 minutes (pre-denaturation), $94{ }^{\circ} \mathrm{C}$ for 1 minute (denaturation); $62{ }^{\circ} \mathrm{C}$ for 45 seconds (annealing); $72{ }^{\circ} \mathrm{C}$ for 2 minutes (extension) in 30 cycles and $72{ }^{\circ} \mathrm{C}$ for 10 minutes (final extension).

\section{Sequence Analysis}

Analysis of $\beta$-actin promoter sequence was performed using TF Bind ${ }^{T M}$ to verify the presence of transcription factor elements which are contained within the sequence (Alimuddin et al. 2008; Kato et al. 2007). DNA sequencing was done at 1 st Base (Singapore). Verification of sequence was conducted by aligning the sequence with Genbank databases of Indian catfish (Heteropneustes fossilis) and yellow catfish (Pelteobagrus fulvidraco) using GENETYX version 7. 


\section{RESULTS AND DISCUSSION}

\section{Genomic DNA Isolation}

The isolation was successfully to obtain the pituitary genomic DNA of C. batrachus on $1 \%$ agarose gel electrophoresis (Figure 1). Based on the size of the marker fragments $1 \mathrm{~kb}$ DNA ladder, DNA containing exons and introns, so the size of the fragments is relatively long. Thus the DNA fragments of the local catfish detected by $1 \%$ agarose gel electrophoresis.

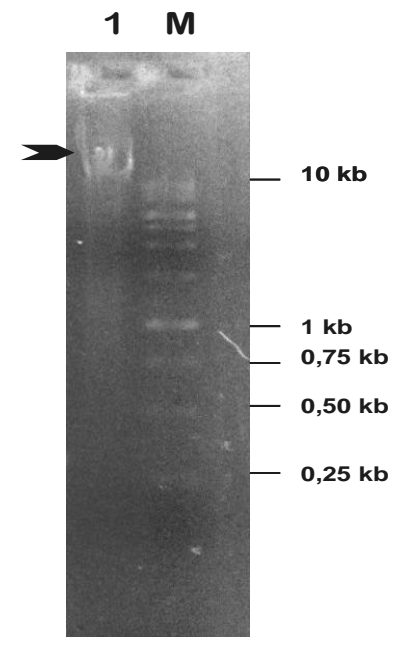

Figure 1. Electroferogram of pituitary genomic DNA of walking catfish, Clarias batrachus ( ) $; 1$ = Pituitary genomic DNA of C. batrachus. $M=1 \mathrm{~kb}$ DNA Marker

\section{PCR Amplification of $\beta$-actin Promoter}

Amplification of $\beta$-actin promoter from pituitary genomic DNA of walking catfish showed an amplicon of $1,7 \mathrm{~kb}$ in length (Figure 2). Ge et al. (2012) also successfully obtained a 1,65 $\mathrm{kb}$ of $\beta$-actin promoter isolated from yellow catfish.

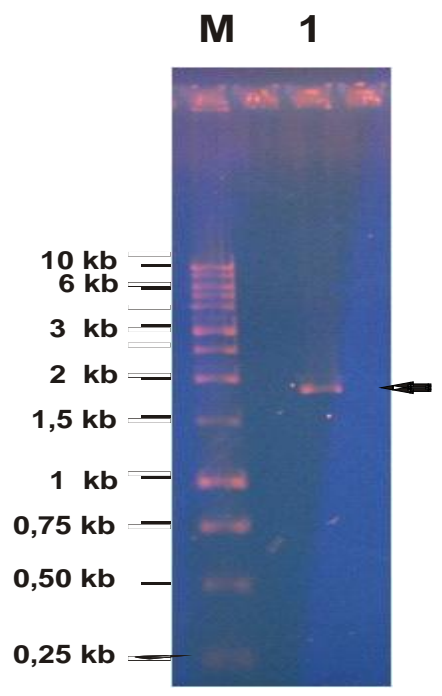

Figure 2. PCR amplification product of $\beta$-actin promoter from pituitary genomic DNA of walking catfish, $C$. bratachus ( $)=\beta$-actin promoter $(1,7 \mathrm{~kb}) . M=1 \mathrm{~kb}$ DNA Marker 


\section{Sequence Analysis}

The sequencing result was showed in Figure 3. The Analysis of transcription factor elements of $\beta$-actin promoter was done using TF Bind ${ }^{T M}$ (Hoffman et al., 1997; Alimuddin et al., 2008). The transcription factor elements of $\beta$-actin promoter are represented by TATA box, CAAT box, and CarG motif (Hew \& Fletcher, 2001). TATA box has important role on transcription regulation in proximal region of promoter, CAAT box is an enhancer which is needed to increase transcription level, and CarG motif is involved in transcription regulation in basal region of promoter (Aranburu et al., 2006; Santoro \& Walsh, 1991). These elements (TATA box, CAAT box, and CarG motif) are found within the sequence of $C$. bratachus $\beta$-actin promoter. The CAAT box was located in nucleotide (nt) of 22-29; TATA box in nucleotide of 49-54 and 296-302; and CarG motif in nucleotide of 615-623.

Other important elements are GC box (GGGCGGG) and TGACG motif, which are involved as intermediate factor of pituitary growth hormone (Argenton et al., 1996). The result of alignment of transcription factor elements of $\beta$-actin promoter of $C$. bratachus and Indian catfish ( $H$. fossilis) using GENETYX version 7.0 was showed on Figure 3. TGACG motif of $\beta$ actin promoter of C. bratachus was found at $814-818 \mathrm{nt}$.

C. batrachus.txt 0

H. fossilis.txt

1:CAAACATATCTTGCATTTCCAGACTGAAATCTTGAGAGACAGGTTTTAAGAGGAAAAGTA 60

C. batrachus.txt 0

H. fossilis.txt

61:TTCACAGGGAGGAGCAGCCTGTGCTCTGCTGTGTTTGAATGATCAGATAAGCAGGGGG AC $\quad 120$

C. batrachus.txt

H. fossilis.txt 0

121 :TTGCTCGACAGCTTTATACAGGTTCATATTTTATATTTATTTATATTTGAACTTTGTAAA 180

C. batrachus.txt

H. fossilis.txt

181 :CCTCTATATGTATTAATATTTGTTCAAGAACATATATCTTAGATTAGGCCTCACATCAAT 240

C. batrachus.txt 0

0

H. fossilis.txt

241 :CCCTTTTCCCCTAGCCCGTTACTGTTATCGCCTCCCTCTTTCTACGCTACGCTCAGTGCA 300

C. batrachus.txt TANGNNNNTCTTC 13 
H. fossilis.txt

301 :CCACAGCGTGCACCCGACGTGCCCCAGTGAGTGACGCTGGACCAATCACAGCCGCG ATTC 360

C. batrachus.txt 14:CGAAAAGGGTCCATTT---TAGACGGCCATGT---GGAGCG--TATAAAACCAGGCGCC 64

H. fossilis.txt

361 :CGAAAGTTTACCTTTTATGGAAAGGGCCGGGCAACGGACGGACTATAAATACCACGC CCA 420

C. batrachus.txt 65:CAGACTCCCACTTCACTT-

TGAGCTCCTCCACACGCAGCTAGTGCGGAATATCATCTGCC $\quad 123$

H. fossilis.txt 421:CGGCTAGCAAATTCACTACTGAGCGCCGTCACAC-

CAGCTTGTGCGGA-TATCATTCGCC 478

C. batrachus.txt 124:C--

AACCAAATTTATTTTTTCTTAAGCCGACAACCCCCAAATCTTAAGGTAAGTTTTTTT

H. fossilis.txt 479:TGAAACCGATTCCCTTGAAACTCATGCTT 507

C. batrachus.txt

182:CCCCCTTTCCTCCTGGTATTGTTATTACTGTTAGCAATAGTAATTGCAGTAACAATAGTA

241

H. fossilis.txt

507:

-----------------507

C. batrachus.txt 242:ACATTGCTATTTATGTATGCAAGGGTTTTAATTGTAAAAACTATATATATATTTTTATAA 301

H. fossilis.txt 507

-----------------507

C. batrachus.txt 302:ATTAATGAATGACTGCAAAAAGAACAATACGTTTTCCCTTTATCATGCAGCGATATTAAA 361

H. fossilis.txt 507

507

C. batrachus.txt

362:CTAGACAGGAATTATTTTTGTAAATTTTTACCTCAGGTTTTTTTTTTTTTTTTCCCTGGGG 421

H. fossilis.txt 507
507

C. batrachus.txt 422:GCTAAAAGCCCCGTAAAACGGCGGGGAGGGGGGGGTTTTTTTTTTATTTCTATAAATGAA A 481

H. fossilis.txt 507 507

C. batrachus.txt 482:AAACCGTTTTTTGCCCTGATTATTAAAAAGCGGAGGAAGACCCGTATTTGTCGACGATAA 541

H. fossilis.txt 507 507 
C. batrachus.txt

542:TCACAAAAAAAGCGGGGGCGGGGGGCCTCCCCCGTTTTTTCCACCTCCCCCCCGGAGA GG 601

H. fossilis.txt

507:

507

C. batrachus.txt

602:GTAGGATTTCACCCAAATTGGGACAACGAGACCCCTGGGGTAATTCGCTGCCTACCCC TG 661

H. fossilis.txt 507

507

C. batrachus.txt

662:GGCACTTGAAAAGGGGGGCCCAGTCGTGCTTAAGGCTGTCAAGCCAAGAAGGCTCC CCCA 721

H. fossilis.txt 507:507

C. batrachus.txt

722:TTTTTTTCTCGCTAGGGGGGGGGGCCAGAAAAAATAATATATTTTTTTTATTTTATACCT 781

H. fossilis.txt

507:-

507

C. batrachus.txt

782:ACTTCCTCACAACTGTACAAAAAAAAGCCACTGACGAGCTCTTGTTATTACAACACCCAG 841

H. fossilis.txt 507: 507

C. batrachus.txt

842:AGATATTGTCCGTCTTATAATTTTTTTTTCTAAAGGCGCCACAAAGCGGACCACACACTT 901

H. fossilis.txt 507

507

C. batrachus.txt

902:AAATCATAGTTAAGTAAGGCCGCCTTGTTGGTGAGCAAGTATACAGGACCGGGAAGTA GA 961

H. fossilis.txt 507: 507

C. batrachus.txt 962:GATAGAATGAGTTGATAAAATTAAAATATAAATTTGTTCAGNNTGAACGGAGGCCTGCC C 1021

H. fossilis.txt 507 507

C. batrachus.txt 1022:GGGCTCTGCAGAT 1034

H. fossilis.txt

507:

507 
Figure 3. Alignment result of of $\beta$-actin promoter of $C$. bratachus and Indian catfish ( $H$. fossilis, genbank : AY 531754.1) (Meenakumari et al. 2004)

CAAT box $=$ GGCCAATCT
CarG = CC(A/T) ${ }_{6} G G$
TATA box = TATAAA
GC box = GGGCGGG

In the alignment result of $\beta$-actin promoter of $\mathrm{C}$. bratachus and yellow catfish (Pelteobagrus fulvidraco) also indicate these transcription factor elements (TATA box, CAAT box, and CarG motif) (Figure 4).

C. batrachus.txt yellow catfishtrt 0

1:GTGAGTGCGCCGGACCAATCAGACGAAGCGATGCCGAAAGTTTACCTTATATGGAAGTT G 60

C. batrachus.txt 0

yellow catfish.txt

0

61:CCGGCCACAGTGCCGGGTATAAATACAGCGTCGCCCCGGTTAAGCTGCCACTCTGAGT TT 120

C. batrachus.txt 0

yellow catfish.txt

121 :GCCTGTGCACGAGTCTAGAAGGACATTAATTCCAGCTATATTCCTGTTGAACCACTGATT 180

C. batrachus.txt

$$
0
$$

yellow catfish.txt

181:CTTTGGTAAGATCAGCTTTATCTTTGTCTACATACGATTGTGATTATAACTACATCCTCT 240

C. batrachus.txt $1:-----------------$

TANGNNNNTCTTCCGAAAAGGGTCCATTTTAGACGGCCATGT

42

yellow catfish.txt

241:TTCTACCTTAAATCTATTTTTATTTGTTTTATTAACGATGTACCTGATTTATATCGAGTT 300

C. batrachus.txt

43:GGAGCGTATAAAACCAGGCGCCCAGACTCCCACTTCACTTTGAGCTCCTCCACACGCA GC 102

yellow catfish.txt 301:GG--CGTTTATTTCAGTGCTTCC---

CTGTACATGAGCTCTGGGGTTATGATGACGTTAT 355

C. batrachus.txt 103:TAGTGCGGAATATCATCTGCCCAACCAAATTTATTTTTTCTTAAGCCGACAACCCCCAA 161 yellow catfish.tXt 356:TAATGTGTTCTATAATGATTTATAGAGAGATTCGTTACTCAGTGTAAGGAGAAACTG—A 413 
C. batrachus.txt

162:ATCTTAAGGTAAGTTTTTTTCCCCCTTTCCTCCTGGTATTGTTATTACTGTTAGCAATAG

221

yellow catfish.txt

41 4:GACAATACAAAGATTTATTAATACAGTGAGTGTGTGTGTGTGTGTGTGTGTGTGTGTGTG 473

C. batrachus.txt

222:TAATTGCAGTAACAATAGTAACATTGCTATTTATGTATGCAAGGGTTTTAATTGTAAAAA

281

yellow catfish.txt 474:TGTGTGT-GTGAGAGATG---

CTGTGGTGTTTAAGTTAGATGTGGGTGTGGCTGGTGTTG

C. batrachus.txt

282:CTATATATATATTTTTATAAATTAATGAATGACTGCAAAAAGAACAATACGTTTTCCCTT

341

yellow catfish.txt 530:ATGCAGCT-

TAGCTTCAGTTTGTCTTCAGAAACATTATAATGAAGTTTGCATGATTAATT

588

C. batrachus.txt

342:TATCATGCAGCGATATTAAACTAGACAGGAATTATTTTTGTAAATTTTTACCTCAGGTTT

401

yellow catfish.txt 589:TACC--TCAGTGAAATTAATTAATTTCTGGATTAAAAAAA-

AACCTGTTAAGTTATACAG 645

C. batrachus.txt

402:TTTTTTTTTTTTCCCTGGGGGCTAAAAGCCCCGTAAAACGGCGGGGAGG----GGGGGG $\quad 456$

yellow catfish.txt 646:TAAAGCTACAGTTGCTGCAG-

TAAATTAAACATGATTTGGTGAGCAGGTTTTTGGAGAG 703

C. batrachus.txt

457:TTTTTTTTTATTTCTATAAATGAAAAAACCGTTTTTTGCCCTGATTATTAAAAAGCGGAG 516

yellow catfish.txt 704:CAGGTTTTTTTGTGTACAA-TGTGGGACTCGATCATGGACCC---

TTCAGCAGGGCGCAT 759

C. batrachus.txt 517:GAAGACCC-GTATTTGTCGACGA-

TAATCACAAAAAAAGCGGGGGCGGGGGGCCTCCCCC 574

yellow catfish.txt

760:AATGAGGCTGTAAAAAAAGGGGAGTGGTCGCGTCTCACGAGCCGGTCGAACTTATTA

CCA 819

C. batrachus.txt

575:GTTTTTTCCACCTCCCCCCCGGAGAGGGTAGGATTTCACCCAAATTGGG-

ACAACGAGAC 633

yellow catfish.txt

820:TATAAGGCAATAGCGAGTCTGTTGGCCACTTCCTTTGTCTCAAACCGCCCAGTTTTTGGG 879

C. batrachus.txt 634:CCCTGGGGTAATTCGCTGCCTACCCCTGGGC--------ACTTGAA-AAGGGGG-GCC 681

yellow catfish.txt

880:CTTTGCGCATGTGCGCCACCTGCTGGTGATATATATATTTTTTTAAATAAAGGGTGTGTG 939 
C. batrachus.txt 682:CAGTCGTGCTTAAG-

GCTGTCAAGCCAAGAAGGCTCCCCCATTTTTTTCTCGCTAGGGG 739

yellow catfish.txt

940:CATTCCTACAGCAGATGCTGAATTACCCCTTGTTCTAAATGACCCTGGTCACTTAACTCC 999

C. batrachus.txt

740:GGGGGGCCAGAAAAAATAATATATTTTTTTTATTTTATACCTACTTCCTCACAACTGTAC 799

yellow catfish.txt 1000:TTTGTGTTCTCCACAGCCATGGATGATGAA-

ATTGCCGCACTGGTTGTTGACAACGGATC 1058

C. batrachus.txt 800:AAAAAAAAGCCACTGACGAGCTCTTGTTATTACAACACCCAGAGATATTGTCCGTCTTA 858

yellow catfish.txt 1059:CGGTATGTGCAAGGCTGGATTCGCTGGAGATGATG-

CTCCCCGTGCTGTCTTCC--CATC 1115

C. batrachus.txt

859:TAATTTTTTTTTCTAAAGGCGCCACAAAGCGGACCACACACTTAAATCATAGTTAAGTAA 918

yellow catfish.txt 11 16:CATTGTTGGTCGCCCAAGACACCAGGTA-CGAACCACACAATC--TGCCTCTTATGGAA 1171

C. batrachus.txt

919:GGCCGCCTTGTTGGTGAGCAAGTATACAGGACCGGGAAGTAGAGATAGAATGAGTT GATA 978

yellow catfish.txt 1172:G-CTTAATTGTT--TTAACTATTTTAAATATTATTTAA-

AAACCAAGGGTGCTCAAACT 1226

C. batrachus.txt

979:AAATTAAAATATAAATTTGTTCAGNNTGAACGGAGGCCTGCCCGGGCTCTGCAGAT---1034

yellow catfish.txt

1227:ATTTTTAAGGATAACCTTGTTAGGTTTGAACAATGGCACAACCTTCATCATAGGAGCTTG 1286

Figure 4. Alignment result of of $\beta$-actin promoter of $C$. bratachus and yellow catfish (Pelteobagrus fulvidraco, genbank : EU 161065.2) (Song et al., 2012)

CAAT box $=$ GGCCAATCT

CarG $=\mathrm{CC}(\mathrm{A} / \mathrm{T})_{6} \mathrm{GG}$

TATA box = TATAAA

GC box = GGGCGGG

In this alignment, CAAT boxes are found at nucleotide 35-45 (C.batrachus) and at nucleotide 16-19 (P. fulvidraco); CarG motifs are found at nucleotide 615-623 (C.batrachus) and at nucleotide 1232-1241 (P. fulvidraco); and then, TATA boxes are found at nucleotide 49-54; 296-302; 472-477 (C.batrachus) and at nucleotide 78-83 (P. fulvidraco). As additional, TGACG motifs are found at nucleotide 814-818 (C.batrachus) and at nucleotide 348-352 ( $P$. fulvidraco). While, GC box is found only in C.batrachus at nucleotide 558-564. The presence of these elements (TATA box, CAAT box, and CarG motif) suggested that most likely the isolated sequence is C.batrachus $\beta$-actin promoter. Further more $\beta$-actin promoter of 
walking catfish can be used as a regulator of the expression of protein encoding genes in the production of transgenic fish (Fletcher \& Hew, 2011).

\section{CONCLUSION}

Based on the results of the research, the conclusions are: The $\beta$-actin promoter of C.batrachus is successfully obtained from pituitary genomic DNA by in-vitro amplification (PCR) using $\mathrm{pBAYC}-\mathrm{F}$ dan $\mathrm{PBAYc}-\mathrm{R}$ primers and the transcription factor elements of $\beta$-actin promoter of C.batrachus are represented by the presence of TATA box, CAAT box, and CarG, and also TGACG and GC box.

\section{Acknowledgement}

The Author would like to thank to The Chairman of LPPM-UNPAD who lets this research is funded by Research Grant of "DIPA BLU UNPAD of 2013", also to all of the team members.

\section{REFERENCES}

Alam MS, Lavender FL, lyengar A, Rahman MA, Ayad HH, Lathe R, Morley SD and Maclean N. 1996. Comparison of the activity of carp and rat $\beta$-actin gene regulatory sequences in tilapia and rainbow trout embryos. Mol. Reprod. Dev., 45:47-1 22.

Alimuddin. 2003. Introduction and expression of foreign $\Delta 6$-desaturase-like gene in a teleostean fish. Thesis. Tokyo University of Fisheries, Japan.

Alimuddin, Yoshizaki, G., Kiron, V., Satoh, S., and Takeuchi, T. 2005. Enhancement of EPA and DHA biosynthesis by over-expression of masu salmon "6-desaturase-like gene in zebrafish. Transgenic Research 14:159-165.

Alimuddin, A. Octavera, O.Z. Arifin dan K. Sumantadinata. 2008. Karakterisasi promoter $\beta$ actin ikan nila (Oreochromis niloticus). Jurnal Akuakultur Indonesia 7(2):115-127.

Aranburu, A., D. Liberg, B. Honore, and T. Leandersen. 2006. CarG box-binding factor-A interacts with multiple motifs in immunoglobulin promoter and has a regulated sub cellular distribution. Eur. J. Immunol. 36:2192-2202.

Argenton, F, S. Bernardini, S. Puttini, M. Bortolessi. 1991. A TGACG motif mediates growthhormone factor-1/pituitary-transcriptional-activator-1-dependent CAMP regulation of the rainbow trout growth hormone promoter. Eur. J. Biochem. 238:591-598.

Ath-thar, M.F. 2007._The effectiveness of a-actin promoter medaka fish (Oryzias latipes) with marker genes hrGFP (humanized Renilla reniformis Green Fluorescent Protein) in catfish (Clarias sp.) FO progeny. Thesis Department of Aquaculture. Faculty of Fisheries and Marine Sciences. Bogor. Bogor Agricultural University.

Fletcher, G.L., R.S. Hobbs, R.P. Evans, M.A. Shears, A.L. Hahn and C.L. Hew. 2011 . Lysozyme transgenic Atlantic salmon (Salmo salar L.).Aquaculture Reseacrh 42:427-440.

Ge JZ. Dong, J. Li, Z. Xu, W. Song, J. Bao, D. Liang, and J. Li. 2012. Isolation of yellow catfish $\beta$-actin promoter and generation of transgenic yellow catfish expressing enhanced yellow flourescent protein. Transgenic Research $21(5)$ : $99-1004$.

Hew CL., and G.L. Fletcher. 2001. The role of aquatic biotechnology in aquaculture. Aquaculture 197:191-204.

Higashijima, S., H. Okamoto, N. Ueno, Y. Hotta and G. Eguchi. 1997. High frequency generation of transgenis zebrafish which reliably express GFP in whole muscles or the whole body by using promoters of zebrafish origin. Dev. Biol. 192:289-299.

Hoffman, A., T. Oelgeschlager and R.G Roeder. 1997. Review considerations of transcriptional central mechanism: Do TFIID-core promoter complexes recapitulate nucleosome-like functions. Prot.Natl.Acad.Sci. USA 94:8928-8935. 
Kato, K., M. Takagi, Y. Tamaru, S-I, Akiyama, T. Konishi, O. Murata, and H. Kumai. 2007. Construction of an expression vector containing a $\beta$-actin promoter region for gene transfer by microinjection in red se bream Pagrus major. Fisheries Science 73:440-445.

Kobayashi, S-I, Alimuddin, T. Morita, M. Miwa, J. Lu, M. Endo, T. Takeuchi, and G.Yoshizaki. 2007. Transgenic Nile tilapia (Oreochromis niloticus) over-expression growth hormone show reduced ammonia excretion. Aquaculture 270:427-435.

Maclean, N, and R.J. Laight. 2000. Transgenic fish: an evaluation of benefits and risks. Fin Fish., 1:1 146-172.

Meenakumari, S., R. Priyadharsini, P. Gunasekaran, T.Pandian, and S. Mathavan. 2004 (unpublished). Molecular cloning and characterization of $\beta$-actin promoter from Indian catfish, Heteropneustes fossilis.

Santoro, I.M., and K. Walsh. 1991. Natural and synthetic DNA elements with the CarG motif differ in expression and protein-binding properties. Molecular and Cellular Biology $11(12): 6296-6305$.

Song, W., L.X. Wang, and Q.S. Zhao. 2012 (unpblished). Isolation of yellow catfish $\beta$-actin and generation of transgenic yellow catfish expressing enhanced yellow flourescent protein. 